Technical note

\title{
Positioning accuracy of a single-isocenter multiple targets SRS treatment: A comparison between Varian TrueBeam CBCT and Brainlab ExacTrac
}

\author{
Eliane Graulieres *, Samuel Kubler, Elodie Martin, Régis Ferrand \\ Department of Medical Physics, Cancer University Institute - Oncopole, Toulouse, France
}

\section{A R T I C L E I N F O}

\section{Keywords:}

Multiple targets

SRS treatment

Image-guided radiation therapy

Positioning accuracy

\begin{abstract}
A B S T R A C T
Purpose: This study compared the positioning accuracy between cone-beam CT (CBCT) and ExacTrac (ETX) for a single-isocenter multiple target stereotactic radiosurgery (SRS) on two TrueBeam STx systems.

Methods: A single-isocenter treatment plan was simulated on an anthropomorphic head phantom with six spherical steel ball bearings (BBs). One of the BBs was chosen to be the isocenter. The five off-isocenter targets were located at various distances from the isocenter. MV portal images were generated to evaluate the deviations between the expected and the real center of the targets after CBCT and ETX positioning, respectively.

Results: The evaluation of the positioning accuracy for the isocenter target showed that CBCT and ETX positioning provided comparable, sub-millimetric results. Deviations in positioning accuracy were also calculated for all other targets, also showing comparable results for CBCT and ETX. Moreover, our study showed that the deviation between CBCT and ETX positioning were in better agreement for TBSTx1 and deviated slightly higher on TBSTx2 (maximum: $1.23 \mathrm{~mm}$ at S/I direction), due to a less perfect alignment between the CBCT coordinate system and the ETX coordinate system on TBSTx2 compared to TBSTx1. This study also showed a correlation between the target positioning accuracy and the distance to the isocenter.

Conclusion: The positioning accuracy of ETX and CBCT for targets located at isocenter and off-isocenter locations was compared on two treatment machines and found comparable. Our study highlights the importance of a proper calibration procedure, to ensure correct alignment between the CBCT, ETX and machine coordinate systems.
\end{abstract}

\section{Introduction}

Whole-brain radiation therapy is no longer the standard upfront treatment for multiple brain metastases [1] due to the associated deterioration in neurocognitive functions [2]. Stereotactic radiosurgery (SRS) or fractionated stereotactic radiotherapy can be used for multiple brain metastases patients with good prognosis [3]. Historically, linear accelerator (linac) arc-based SRS have used multiple isocenters (one per target) for treating brain metastases, but this multiple-isocenter approach is time consuming and dosimetrically sub-optimal. The Gamma Knife system (GK; Elekta AB, Sweden) is designed for SRS of intracranial targets [4].

However, treatment times with GK is also proportional to the number of targets, as well as depend on the number of shots needed for an individual target and decay of Cobalt- 60 sources. Recently, new treatment planning techniques have emerged based on Dynamic Conformal Arcs [6] (DCA) and Volumetric Modulated Arc Therapy (VMAT) [5] for treating multiple brain metastases with a single isocenter. Before treating SRS on a linac, the linac and image-guided system should be verified by a Winston-Lutz (WL) test [7]. However, the WL test is insufficient to ensure positioning accuracy for off-isocenter targets, e.g. when treating multiple metastases with a single isocenter.

In our institution, 120 SRS treatments are delivered each year with two TrueBeam STx linac systems version 2.5 (TBSTx1 and TBSTx2) (Varian Medical System, USA), equipped with PerfectPitch 6D couches and ExacTrac version 6.2.2 (ETX) (Brainlab AG, Germany).

The goal at our institution is to implement mono-isocentric treatments for multiple metastases, while ensuring that PTV margins are not increased for those treatments. Both CBCT and ETX systems are available on our TBSTx1 and TBSTx 2 machines, respectively. CBCT is based on volumetric imaging (3D) while ETX utilizes two oblique X-ray (2D) images. The correlation between ETX and CBCT for positioning accuracy has been described previously in the literature. Kim et al. published that both the ETX and the CBCT systems showed approximately $1 \mathrm{~mm}$

\footnotetext{
* Corresponding author.

E-mail address: graulieres.eliane@iuct-oncopole.fr (E. Graulieres).
} 


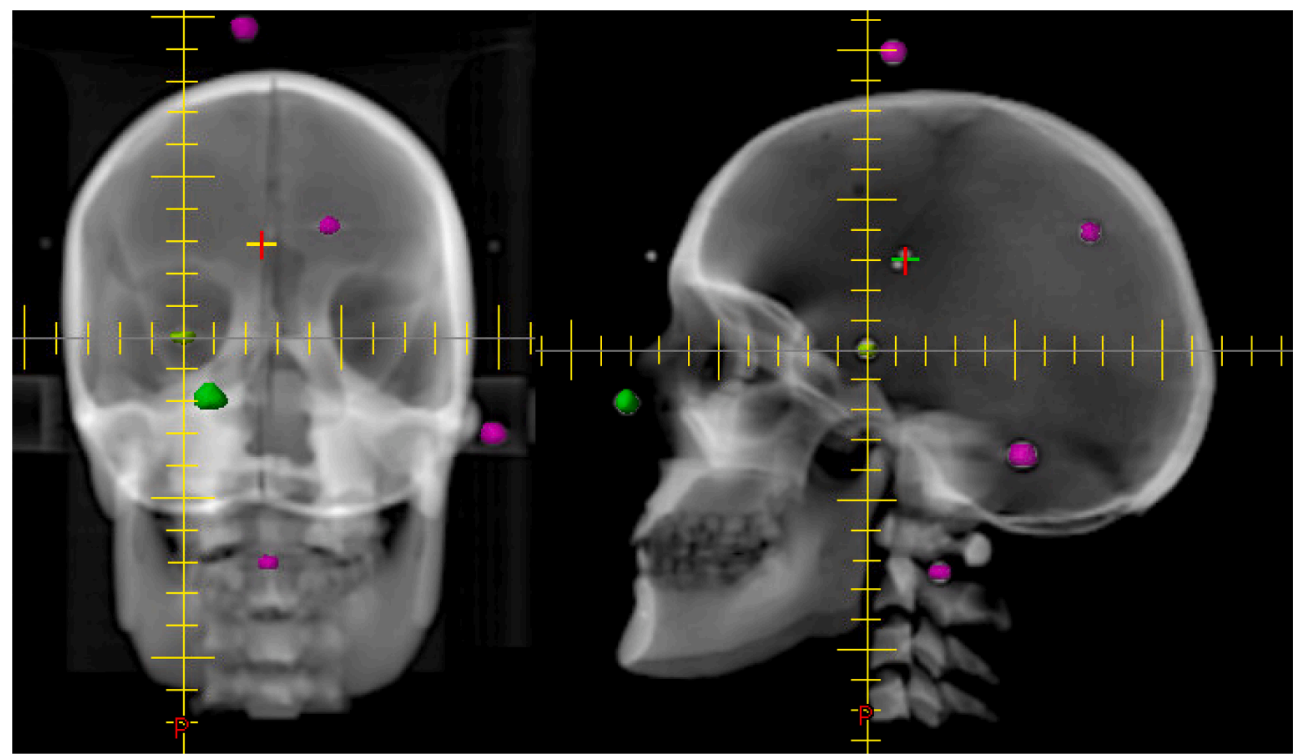

Fig. 1. Coronal and sagittal DRRs of the anthropomorphic head phantom showing the positions of the BBs.

isocenter localization accuracy [8]. Li et al. tested different isocenter locations in a Rando head phantom and found average residual error differences less than $0.4 \mathrm{~mm}$ between ETX and CBCT on a TBSTx [9]. The aim of this study was to compare the positioning accuracy of the CBCT with ETX for a single-isocenter multiple target SRS treatment.

\section{Materials and methods}

\subsection{Phantom}

Six spherical 5-mm diameter steel ball bearings (BBs) to represent six lesions were embedded in an anthropomorphic head phantom (The Phantom Laboratory, Salem, NY, USA). The phantom was scanned on a GE Optima CT580 Scanner with a $1.25 \mathrm{~mm}$ slice thickness. The locations of BBs were chosen to avoid overlapping in portal images. Fig. 1 shows the BBs positions on a coronal and sagittal digital reconstructed radiograph (DRR).

One $\mathrm{BB}$ was chosen as the isocenter (BB0). The $3 \mathrm{D}$ distances between $\mathrm{BBs}$ and the isocenter are in $\mathrm{cm}$ :

BB1: 8.06
BB2: 8.27

BB3: 9.48

BB4: 9.97

BB5: 11.7

\subsection{Treatment planning}

First, the ETX and CBCT isocenter positioning accuracy was investigated using a "hidden target" test [10]. The BB0 was contoured in the treatment planning system (TPS) Eclipse (Varian Medical System, USA) and considered as the Planning Target Volume (PTV) and the isocenter was defined as the center of gravity of BB0. Eight reference setup fields using $2 \times 2$ MLC fields sizes centered on BB0 were created to evaluate setup errors using MV after CBCT positioning and ETX positioning. The MV portal images were acquired at the four cardinal gantry angles $\left(0^{\circ}, 90^{\circ}, 180^{\circ}, 270^{\circ}\right)$ with the collimator at 90 and $270^{\circ}$ for each gantry position.

Second, a single isocenter SRS treatment plan for six brain metastases was simulated. Each BB was contoured as a target and the isocenter of the irradiation plan was positioned at the center of gravity of BB0. The

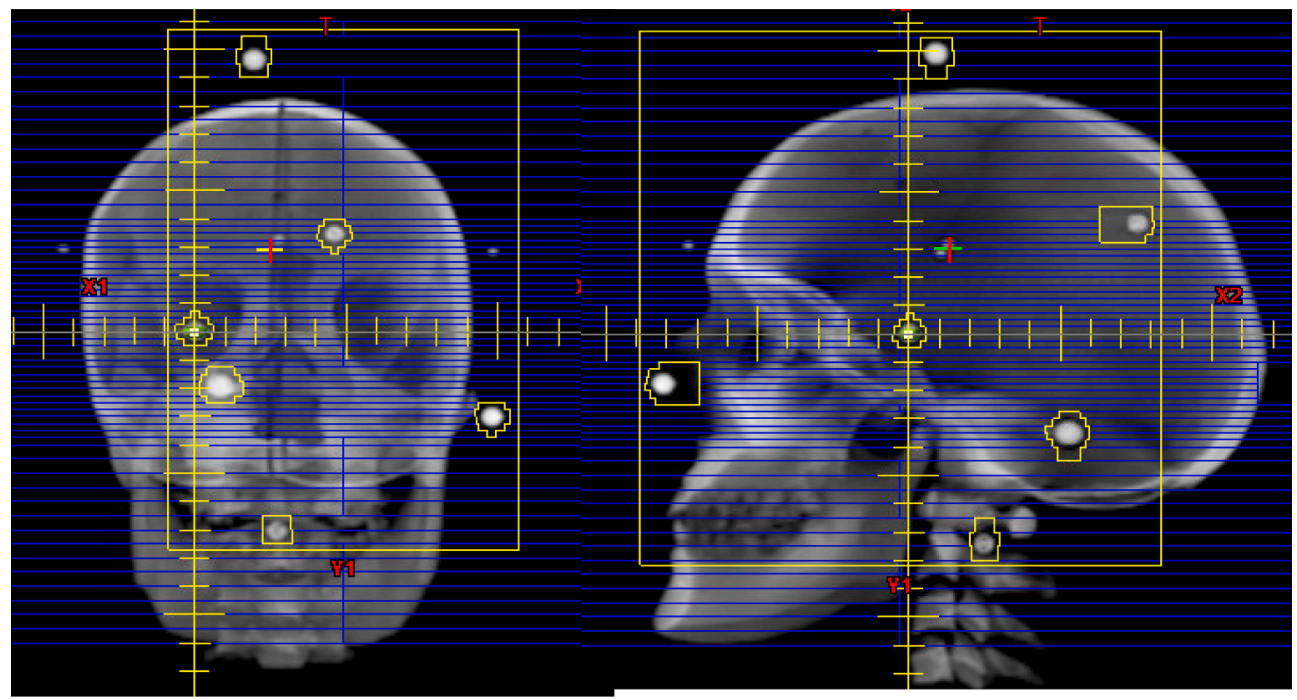

Fig. 2. DRRs of the MV portal image with MLC field for a gantry angle of $0^{\circ}$ and $90^{\circ}$ for the non-isocentric positioning accuracy evaluation. 


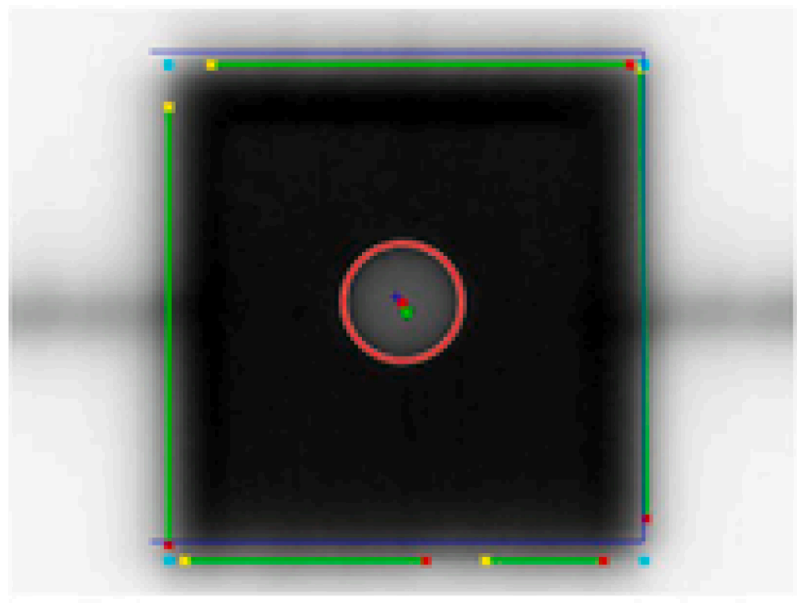

Fig. 3. MV portal image. In red, border and center of BB. In green, border and center of irradiation field. In blue, border and center of field as per Dicom coordinates. (For interpretation of the references to colour in this figure legend, the reader is referred to the web version of this article.)

MLC was fitted around each target with a $2 \mathrm{~mm}$ beam margin around the target. Four reference setup fields, one for each cardinal gantry angle with collimator at $0^{\circ}$, were created to perform the "hidden target" test using MV portal images.

Fig. 2 shows the DRRs with the MLC field for a gantry angle of $0^{\circ}$ and $90^{\circ}$.

Third, the same test was repeated with non-coplanar couch angles commonly used for our SRS treatments: $30^{\circ}, 90^{\circ}$ and $300^{\circ}$. For each couch angle, the phantom was positioned using only ETX. A MV portal image was instead acquired for these couch angles. Collimator angles were chosen to avoid MLC overlapping on a target.

\subsection{Setup and irradiation}

This setup and irradiation procedures were performed for each TBSTx respectively, with an ETX calibration between the first and the second measurement. The motion precision of the PerfectPitch couch is $0.1 \mathrm{~mm}$.

Both ETX and CBCT were previously calibrated to the machine radiation isocenter. An IsoCal calibration [11] was performed to calibrate the CBCT. The Winston-Lutz [7] test was thereafter performed with a pointer to calibrate ETX to the radiation isocenter.

The head phantom was positioned on the $6 \mathrm{D}$ couch using the frameless SRS universal couch extension. The ETX infrared optical positioning system was used to preposition the head phantom. A CBCT to planning-CT registration was then performed.

All the CBCT data were acquired using the standard-dose head protocol $\left(100 \mathrm{kVp}, 150 \mathrm{mAs}, 25 \times 25 \mathrm{~cm}^{2}\right.$ field of view) reconstructed with $1 \mathrm{~mm}$ slice thickness.

For the first test the phantom was prepositioned with the ETX infrared optical positioning system before an online correction with either CBCT or ETX. CBCT images and planning CT were automatically registered. ETX images were also automatically registered to planningCT DRRs, but the BBs were not taken into account during registration. Once online 6D corrections were sent to the treatment couch, either by CBCT correction or ETX correction, the residual setup error was measured using eight MV portal images as mentioned above. The portal image pixel size is $0.261 \times 0.261 \mathrm{~mm}^{2}$ at a source to panel distance of
$150 \mathrm{~cm}$.

The same setup was used to perform the second test evaluating the positioning accuracy of off-isocenter targets using CBCT and ETX at $0^{\circ}$ couch angle and the third test evaluating the ETX accuracy with noncoplanar couch angles.

\section{Analysis}

\subsection{Positioning accuracy at the isocenter}

The positioning accuracy of the target at isocenter was evaluated with eight MV portal images centered on BB0. The deviations between the center of $\mathrm{BB}$ and the center of the irradiation field were calculated in anterior/posterior (A/P), left/right (L/R) or inferior/superior (I/S) using MATLAB image processing toolbox software (Mathworks, USA).

For each image, the coordinates of the center of the BB and the center of the irradiation field were extracted using a Hough transform [12]. Fig. 3 shows the analysis performed on one of the portal images. The final deviation in each direction was then the average of all the deviations calculated in this direction.

\section{Positioning accuracy for off-isocenter targets at couch $0^{\circ}$}

The positioning accuracy of the off-isocenter targets was determined using four MV portal images. The planned field shape on the DRR image was registered to the irradiation field shape on the MV portal image according to the MLC field borders using a procrustes analysis. For each $\mathrm{BB}$, the center was detected on each image and the deviation between the expected location in the DRR and the measured one on the MV image was then calculated in $\mathrm{A} / \mathrm{P}, \mathrm{L} / \mathrm{R}$ or I/S depending on the gantry angle. The final deviation in each direction is the average of all the deviations estimated from all MV portal images.

\section{Positioning accuracy of targets with non-coplanar couch angles}

The same analysis was performed with the non-coplanar treatment plan. Only MV portal images with a gantry angle of $0^{\circ}$ could be performed. Therefore, the deviations obtained were only in L/R and S/I directions.

\subsection{Statistical analysis}

A Wilcoxon signed rank test was used to test the difference between CBCT and ETX for the off-isocentric target positioning accuracy and between TBSTx1 and TBSTx2 for the non-coplanar positioning accuracy.

\section{Results}

\subsection{Isocenter target positioning accuracy}

Table 1 shows the average and the standard deviation of the deviations $(\Delta)$ in $\mathrm{mm}$ after CBCT and after ETX when looking at the isocenter of the treatment plan for each TBSTx.

The deviations for both TBSTx1 and TBSTx2 were less than $1 \mathrm{~mm}$ after $6 \mathrm{D}$ positioning either by ETX or CBCT. The maximum deviation was $0.58 \mathrm{~mm}$ at the $\mathrm{S} / \mathrm{I}$ direction for TBSTx2. The deviations after CBCT positioning were comparable to those after ETX positioning. Furthermore, the deviations were similar for both TBSTx systems. The maximum vector length was $0.69 \mathrm{~mm}$ after ETX positioning for TBSTx2. 
Table 1

Deviations \pm standard deviation in mm after CBCT and ETX positioning for TBSTx1 and TBSTx2 between the center of BB and the center of the irradiation field.

\begin{tabular}{|c|c|c|c|c|c|c|c|c|}
\hline & \multicolumn{4}{|l|}{ TBSTx1 } & \multicolumn{4}{|l|}{ TBSTx2 } \\
\hline & $\Delta \mathrm{L} / \mathrm{R}$ & $\Delta \mathrm{A} / \mathrm{P}$ & $\Delta \mathrm{S} / \mathrm{I}$ & Vector length & $\Delta \mathrm{L} / \mathrm{R}$ & $\Delta \mathrm{A} / \mathrm{P}$ & $\Delta \mathrm{S} / \mathrm{I}$ & Vector length \\
\hline CBCT positioning & $0.13 \pm 0.40$ & $-0.06 \pm 0.32$ & $-0.40 \pm 0.21$ & $0.43 \pm 0.55$ & $-0.01 \pm 0.13$ & $-0.02 \pm 0.08$ & $-0.18 \pm 0.23$ & $0.18 \pm 0.28$ \\
\hline ETX positioning & $0.05 \pm 0.32$ & $0.03 \pm 0.29$ & $-0.51 \pm 0.21$ & $0.51 \pm 0.48$ & $0.17 \pm 0.11$ & $0.33 \pm 0.14$ & $-0.58 \pm 0.22$ & $0.69 \pm 0.28$ \\
\hline
\end{tabular}

\section{Off-isocenter target positioning accuracy}

Table 2 shows the average and the standard deviation of the deviations $(\Delta)$ in mm after CBCT and after ETX for each target as a function of distance from the isocenter in $\mathrm{cm}$ for the coplanar treatment plan for each TBSTx.

The deviations were calculated for the six BBs. The deviations were less than $1 \mathrm{~mm}$, except for TBSTx1 after CBCT positioning at S/I direction and for 4 values of deviation for TBSTx 2 after ETX positioning. The maximum deviation found was $1.2 \mathrm{~mm}$ for TBSTx2.

The deviations in the S/I direction were more important in all cases except for TBSTx2 after CBCT positioning. After CBCT positioning, they were comparable between TBSTx1 and TBSTx 2 and were also comparable to TBSTx1 after ETX positioning.

For TBSTX2, the deviation (vector length) was always (except once) superior with ETX registration compared to CBCT registration.

Fig. 4 reports the deviations represented by the vector length for each method and each machine as a function of the distance from the isocenter. A linear trend for each plot is also provided.

Scatter plots of TBSTx1 and TBSTx2 were close after CBCT positioning as well as TBSTx1 after ETX positioning. The scatter plot for TBSTx2 was larger than the others (except for one point) with a maximum vector of length of $1.58 \mathrm{~mm}$ for BB5. The difference between CBCT and ETX was statistically significant for TBSTx2 showing that CBCT gave better results ( $\mathrm{p}<0.0001$ ). For all methods, the correlation coefficient was positive. The general trend was the greater the distance, the greater the deviation, although some deviations measured did not follow the trend. Coefficients of determination were also generated for the four curves. The calculated coefficient for TBSTx1 after CBCT positioning $\left(\mathrm{R}^{2}=0.83\right)$ showed a good correlation between the deviations measured on TBSTx1 after CBCT positioning and distance from isocenter. This correlation is positive but less important for ETX.

\section{Non-coplanar positioning accuracy}

Table 3 shows the average and the standard deviation of the deviations $(\Delta)$ in mm after ETX for each target in function of the distance from isocenter in $\mathrm{cm}$ for the non-coplanar treatment plan.

The deviations were only measured at $\mathrm{L} / \mathrm{R}$ and $\mathrm{S} / \mathrm{I}$ directions as a single portal image at gantry angle $0^{\circ}$ was acquired for non-coplanar couch angles. The maximum deviation for TBSTx1 was $1.08 \mathrm{~mm}$ for BB2. It was $1.33 \mathrm{~mm}$ for TBSTx2 for the same BB. Vector lengths were also calculated. The maximum vector length for TBSTx 1 was $1.32 \mathrm{~mm}$ for BB5. It was $1.78 \mathrm{~mm}$ for TBSTx2 for. Deviations on TBSTx1 and TBSTx2 were close.

\section{Discussion}

The aim of this study was to investigate the ETX and CBCT positioning accuracy via "hidden target" tests. Before performing the tests, the CBCT system was calibrated with the IsoCal procedure and ETX system was calibrated with a Winston-Lutz pointer. Du et al. found that the offset between the IsoCal-calibrated image centers and the WinstonLutz radiation isocenter is within $0.4 \mathrm{~mm} \mathrm{[13]}$ and we assumed that the isocenters of the CBCT and the ETX system were correctly calibrated. These calibrations were performed before each test in order to ensure a good agreement between both imaging systems.

First, the test for the isocentric positioning accuracy was performed. The isocenter of the irradiation was the center of gravity of the BB0. The deviations between the center of the $\mathrm{BB}$ and the center of the irradiation field measured after positioning by ETX and positioning by СВCT were similar for both machines. The maximum vector length was $0.69 \mathrm{~mm}$. Kim et al. also found that the isocenter variation for both ETX and CBCT was in the range of $1 \mathrm{~mm}$ [8]. Oh et al. did a study based on residual setup errors between ETX and CBCT on 107 patients [14]. They showed that the root-mean-square of the difference in the translational dimensions between ETX and СВCT was $1.01 \mathrm{~mm}$ for online matching. They concluded that the discrepancies in residual setup errors were minor but should not be ignored. Chang et al. had the same conclusion [15]. For target at isocenter positioning accuracy, we did not find any significant difference between both systems. This is in accordance with the study of Huang et al. [16] and Li et al [9].

Secondly, the off-isocenter target positioning accuracy was performed, where most of the deviations were found to be less than $1 \mathrm{~mm}$. Ezzel et al. found that for distances of $10 \mathrm{~cm}$ or more, the CBCT-based alignment was within $1.1 \mathrm{~mm}$ while the ETX-based alignment differed by up to $2.2 \mathrm{~mm}$ [17] on a Varian iX linac. We did not find such difference, but our studies were carried out on a TBSTx more accurate than a iX linac $(+/-1 \mathrm{~mm}$ vs $+/-0.5 \mathrm{~mm})$. Notably, their phantom was made of three sections of wood without any anatomy for X-ray registrations, while we used an anthropomorphic phantom Our study was consistent with the one of Zollner et al. [18]. They found that the two imaging modalities (XVI/CBCT and ETX) were in close agreement on a scale of about one millimeter on an Elekta Versa HD linac. In the same way, Kim et al. found that the positional variations of the off-isocenter targets were less than $1 \mathrm{~mm}$ on a Novalis Tx Linac [19].

Our study showed that deviations between both image-guided systems were close after CBCT and ETX positioning on TBSTx 1 and slightly higher on TBSTx 2 after ETX positioning with a significant statistical difference. In order to understand this discrepancy, we performed the following test: using the same phantom, we made long-range movements $(10 \mathrm{~cm})$ and asked both imaging systems (CBCT and ETX) to "find" the performed motion. Although on TBSTx1, the motion measured by both imaging systems and the motion performed matched within $0.5 \mathrm{~mm}$ and $0.2^{\circ}$ (with a mean value over the tests close to $0^{\circ}$ for each axis), we found on TBSTx2 a slight discrepancy between ETX coordinate system and CBCT coordinate system (rotation of one coordinate system compared to the other around the vertical axis of about $0.35^{\circ}$ ). We confirmed this by performing various longitudinal shifts $(5 \mathrm{~cm}, 10$ $\mathrm{cm}$ ) with a lateral correction increasing linearly with the shift on TBSTx2. This indicates that the ETX coordinate system was less perfectly aligned, although within tolerances, with the CBCT coordinate system 


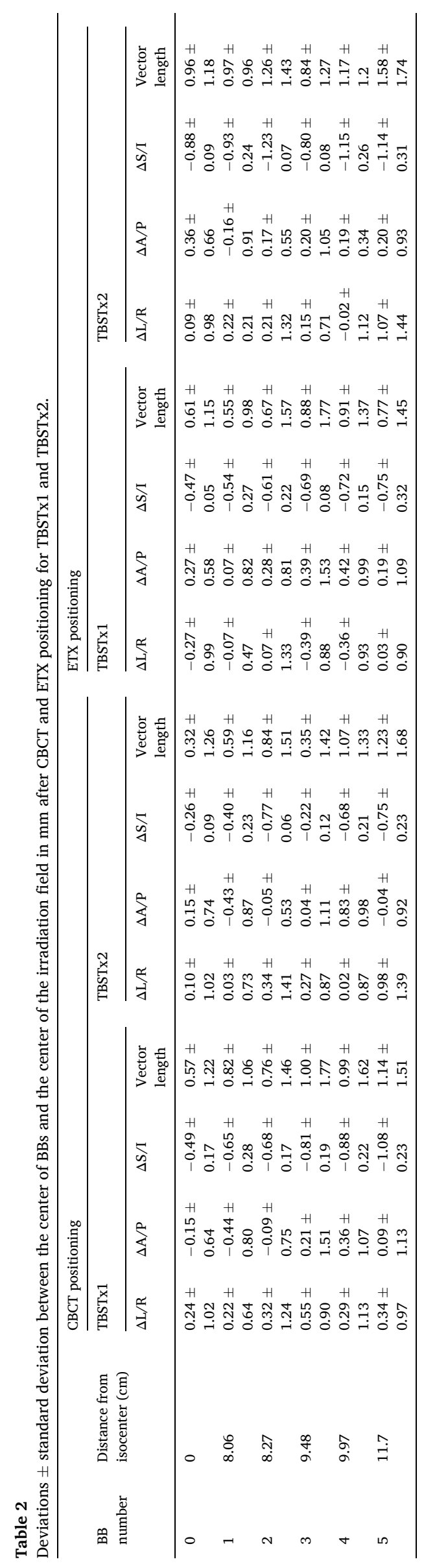

on TBSTx2 compared to TBSTx1. Hence, we have decided to check it quarterly in our institution on both systems by performing these longrange motions (translation and rotations). For very small movements and a single isocenter treatment, this slight misalignment between ETX and CBCT has no consequence. However, with the aim of treating multiple metastases with a single isocenter, careful attention should be paid to the alignment of ETX, CBCT and machine coordinate systems (and not only isocenters, like Isocal or WL tests do). If so, with precise calibration of the ETX coordinate system with respect to the machine coordinate system, the accuracy of ETX (which is based on 2x2D images) is remarkably comparable to these of CBCT (based on 3D matching). This is interesting as for non-coplanar angles, ETX remains the only imaging system to provide $6 \mathrm{D}$ corrections.

With reference to Fig. 4, we can see that there is a positive correlation between the deviations and the distance from isocenter. This means that the greater the distance from the isocenter, the greater the deviation with imaging positioning. Tominaga et al. study using a Varian Novalis $\mathrm{Tx}$ found that the accuracy of off-isocenter target positioning was worse than at the isocenter [20]. In our study, this trend seems to be less obvious after ETX positioning when looking at the correlation between the deviations and the distance from the isocenter.

The maximum vector length found in our study was $1.58 \mathrm{~mm}$ for a BB at a distance of $11.7 \mathrm{~cm}$ from the isocenter. In our institution, the PTV margin for intracranial targets is $2 \mathrm{~mm}$. We still are consistent with the results found in our study if we want to treat single-isocenter multipletargets SRS. However, it is obvious that the deviations must be evaluated for each individual machine and each individual imaging system before employing single-isocenter multiple-target SRS.

Finally, we tested the non-coplanar positioning accuracy. The deviations were higher than those measured in off-isocenter targets positioning accuracy with couch at $0^{\circ}$. As our study was performed on a phantom which does not move, this may be due to the inaccuracy of the couch when it moves to non-coplanar angles. This is consistent with the study of Sarkar et al. [21] with the setup margin increased by $1 \mathrm{~mm}$ in non-coplanar geometry. A clear benefit of ETX system for mechanical couch inaccuracies is that these inaccuracies can be corrected for at noncoplanar couch angles. Indeed, it may not be necessary to use large margins for target located at the isocenter of the irradiation field and this is in agreement with Minniti et al. [22], where the authors examined local control as significant outcome for assessing the accuracy of treatment delivery.

No positive correlation was found for non-coplanar positioning accuracy but here, we were limited because only two dimensions of portal imaging are available. We also cannot compare CBCT positioning with ETX positioning as CBCT is not possible for non-coplanar couch positions.

Although providing a comparison between CBCT and ETX with mirrored machines, this study has several limitations: first, the measurements considered distances (2D and 3D) between planned and real BBs locations, without taking into account MLC errors. Second, as the irradiation scheme is complex (multiple arcs, nominally five, including overlap and/or avoidance), the dosimetric impact is not a simple shift of the isodoses corresponding to the measured vectors.

Another limitation is that this study was performed on an anthropomorphic phantom and did not consider patient motion for frameless SRS treatments.

Finally, there is a lack of BBs between 0 and $8 \mathrm{~cm}$ due to the geometry of the phantom. However, the large distances allowed us to evaluate maximized errors for cranial anatomy.

\section{Conclusion}

When multiple brain metastases are treated using a single isocenter, the image-based alignment may lead to higher inaccuracy for offisocenter targets, which could imply decreased target coverage and subsequent irradiation of normal brain. We validated that both ETX and 


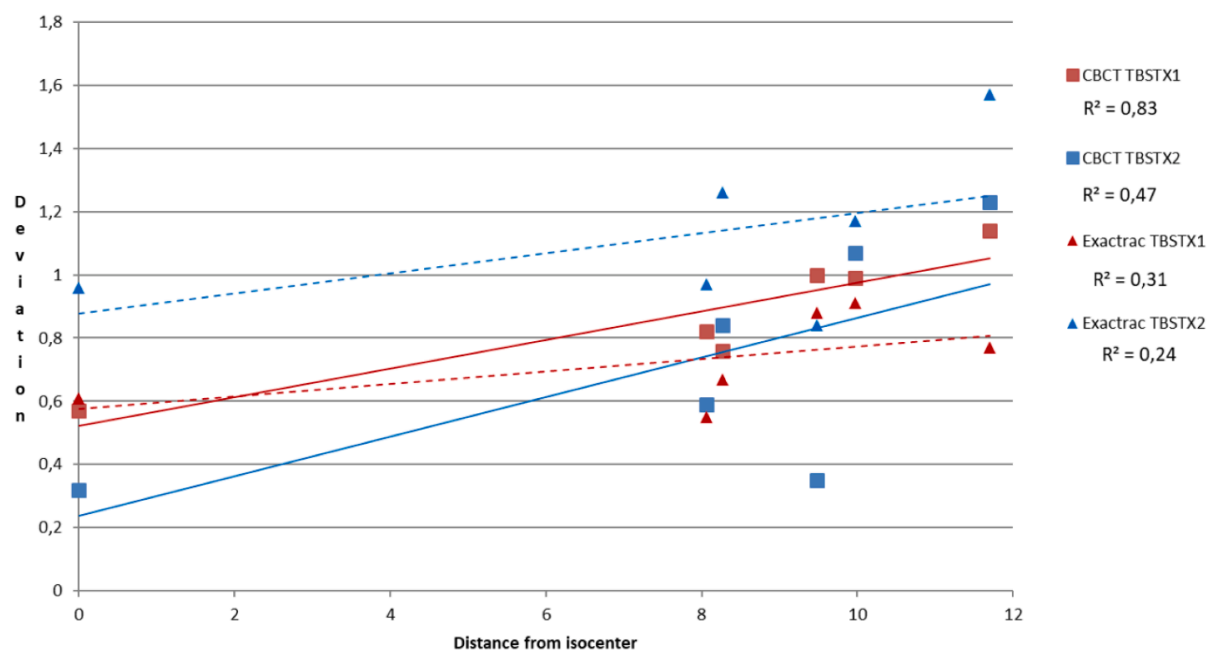

Fig. 4. Deviations in $\mathrm{mm}$ represented by the vector length versus distance from isocenter in $\mathrm{cm}$ for non-isocentric positioning accuracy and linear trend for each set. (Dashed lines : EXT, Full lines : CBCT).

Table 3

Deviations between the center of BBs and the center of the irradiation field in mm after ETX positioning for TBSTx1 and TBSTx2.

\begin{tabular}{|c|c|c|c|c|c|c|c|}
\hline \multirow{3}{*}{ BB number } & \multirow{3}{*}{ Distance from isocenter $(\mathrm{cm})$} & \multicolumn{6}{|c|}{ ETX positioning } \\
\hline & & \multicolumn{3}{|l|}{ TBSTx1 } & \multicolumn{3}{|l|}{ TBSTx2 } \\
\hline & & $\Delta \mathrm{L} / \mathrm{R}$ & $\Delta \mathrm{S} / \mathrm{I}$ & Vector length & $\Delta \mathrm{L} / \mathrm{R}$ & $\Delta \mathrm{S} / \mathrm{I}$ & Vector length \\
\hline 0 & 0 & $0.65 \pm 0.33$ & $-0.97 \pm 0.35$ & $1.16 \pm 0.48$ & $0.52 \pm 0.29$ & $-1.14 \pm 0.44$ & $1.25 \pm 0.53$ \\
\hline 1 & 8.06 & $0.71 \pm 0.23$ & $-1.01 \pm 0.70$ & $1.24 \pm 0.74$ & $0.56 \pm 0.19$ & $-1.00 \pm 0.61$ & $1.15 \pm 0.41$ \\
\hline 2 & 8.27 & $1.08 \pm 0.13$ & $-0.63 \pm 0.67$ & $1.25 \pm 0.68$ & $1.18 \pm 0.49$ & $-1.33 \pm 0.33$ & $1.78 \pm 0.59$ \\
\hline 3 & 9.48 & $0.42 \pm 0.12$ & $-0.93 \pm 0.33$ & $1.02 \pm 0.35$ & $0.10 \pm 0.38$ & $-0.57 \pm 0.52$ & $0.58 \pm 0.64$ \\
\hline 4 & 9.97 & $0.35 \pm 0.23$ & $-0.62 \pm 0.17$ & $0.71 \pm 0.29$ & $0.25 \pm 0.53$ & $-0.70 \pm 0.79$ & $0.74 \pm 0.95$ \\
\hline 5 & 11.7 & $0.78 \pm 0.32$ & $-1.07 \pm 0.72$ & $1.32 \pm 0.79$ & $1.11 \pm 0.61$ & $-0.73 \pm 1.94$ & $1.33 \pm 2.03$ \\
\hline
\end{tabular}

CBCT were in close agreement for positioning the patient for these type of treatments. The accuracy of ETX has been demonstrated comparable to that of CBCT, provided that a precise calibration of the various coordinate system (and not only isocenters) is being performed.

\section{Funding}

This work was supported by Brainlab AG, Germany.

\section{Appendix A. Supplementary data}

Supplementary data to this article can be found online at https://doi. org/10.1016/j.ejmp.2020.10.022.

\section{References}

[1] Tsao MN, et al. Whole brain radiotherapy for the treatment of newly diagnosed multiple brain metastases. Cochrane Database Syst Rev 2018. https://doi.org/ 10.1002/14651858.CD003869.pub4.

[2] Nieder C, Grosu AL, Gaspar LE. Stereotactic radiosurgery (SRS) for brain metastases: a systematic review. Radiat Oncol 2014;9:155.

[3] O'Beirn M, et al. The expanding role of radiosurgery for brain metastases. Medicines 2018;5.

[4] 4. Physics of gamma knife approach on convergent beams in stereotactic radiosurgery. Int. J. Radiat. Oncol. 18, 941-949 (1990).

[5] Alongi F, Fiorentino A, Ruggieri R, Ricchetti F, Kupelian P. Cost-effectiveness of Linac-based single-isocenter non-coplanar technique (HyperArcTM) for brain metastases radiosurgery. Clin Exp Metastasis 2018;35:601-3.

[6] Mori Y, Kaneda N, Hagiwara M, Ishiguchi T. Dosimetric study of automatic brain metastases planning in comparison with conventional multi-isocenter dynamic conformal arc therapy and gamma knife radiosurgery for multiple brain metastases. Cureus 2016. https://doi.org/10.7759/cureus.882.

[7] Du W, Johnson JL, Jiang W, Kudchadker RJ. On the selection of gantry and collimator angles for isocenter localization using Winston-Lutz tests. J Appl Clin Med Phys 2016;17:167-78.
[8] Kim J, et al. Image-guided localization accuracy of stereoscopic planar and volumetric imaging methods for stereotactic radiation surgery and stereotactic body radiation therapy: a phantom study. Int J Radiat Oncol 2011;79:1588-96.

[9] Li J, et al. Comparison of Online 6 Degree-of-Freedom Image Registration of Varian TrueBeam Cone-Beam CT and BrainLab ExacTrac X-Ray for Intracranial Radiosurgery. Technol Cancer Res Treat 2017;16:339-43.

[10] Solberg TD, Medin PM, Mullins J, Li S. Quality assurance of immobilization and target localization systems for frameless stereotactic cranial and extracranial hypofractionated radiotherapy. Int J Radiat Oncol 2008;71:S131-5.

[11] Chiu TD, Yan Y, Foster R, Mao W. Long-term evaluation and cross-checking of two geometric calibrations of kV and MV imaging systems for Linacs. J Appl Clin Med Phys 2015;16:306-10.

[12] Du W, Yang J. A robust Hough transform algorithm for determining the radiation centers of circular and rectangular fields with subpixel accuracy. Phys Med Biol 2009;54:555-67.

[13] Du W, Gao S, Jiang W, Kudchadker RJ. Independent evaluation of the effectiveness of IsoCal in improving image center accuracy on Varian TrueBeam and Clinac machines. J Appl Clin Med Phys 2018;19:483-90.

[14] Oh SA, Park JW, Yea JW, Kim SK. Evaluations of the setup discrepancy between BrainLAB 6D ExacTrac and cone-beam computed tomography used with the imaging guidance system Novalis-Tx for intracranial stereotactic radiosurgery. PLOS ONE 2017;12:e0177798.

[15] Chang Z, et al. 6D image guidance for spinal non-invasive stereotactic body radiation therapy: comparison between ExacTrac X-ray 6D with kilo-voltage conebeam CT. Radiother Oncol 2010;95:116-21.

[16] Huang Y, et al. Targeting accuracy of image-guided radiosurgery for intracranial lesions: a comparison across multiple linear accelerator platforms. Technol Cancer Res Treat 2016;15:243-8.

[17] Ezzell GA. The spatial accuracy of two frameless, linear accelerator-based systems for single-isocenter, multitarget cranial radiosurgery. J Appl Clin Med Phys 2017; 18:37-43.

[18] Zollner B, et al. Stereoscopic X-ray imaging, cone beam CT, and couch positioning in stereotactic radiotherapy of intracranial tumors: preliminary results from a cross-modality pilot installation. Radiat Oncol 2016;11.

[19] Kim S, Tseng T-C, Morrow A. Spatial variations of multiple off-axial targets for a single isocenter SRS treatment in Novalis Tx linac system. J Radiosurgery SBRT 2015;3:287-96. 
[20] Tominaga $\mathrm{H}$, et al. Accuracy of positioning and irradiation targeting for multiple targets in intracranial image-guided radiation therapy: a phantom study. Phys Med Biol 2014;59:7753-66.

[21] Sarkar B, et al. Technical note: rotational positional error corrected intrafraction set-up margins in stereotactic radiotherapy: a spatial assessment for coplanar and noncoplanar geometry. Med Phys 2019;46:4749-54.
[22] Minniti G, et al. Initial experience with single-isocenter radiosurgery to target multiple brain metastases using an automated treatment planning software: clinical outcomes and optimal target volume margins strategy. Adv Radiat Oncol 2020. 